\title{
Contemporary Birth: A Global Overview
}

\author{
Robbie E Davis-Floyd* \\ Department of Anthropology, USA \\ *Corresponding author: Robbie E Davis-Floyd, Department of Anthropology, USA
}

\begin{tabular}{|c|c|}
\hline ARTICLE INFO & ABSTRACT \\
\hline Received: 幽 July 18, 2019 & From the beginnings of our species, birth was exclusively the work of women as they \\
\hline Published: 蔧 August 13, 2019 & labored to push their babies from the private inner world of their wombs into the larger \\
\hline $\begin{array}{l}\text { Citation: Robbie E Davis-Floyd. Con- } \\
\text { temporary Birth: A Global Overview. } \\
\text { Biomed J Sci \& Tech Res 20(3)-2019. } \\
\text { BJSTR. MS.ID.003467. }\end{array}$ & $\begin{array}{l}\text { have their babies pulled through the "birth canal" with forceps or vacuum extractors, or cut } \\
\text { from their wombs via cesarean section. The medical definition of birth is the emergence } \\
\text { of a fetus from a womb-a definition that ignores all issues of women's involvement and } \\
\text { agency. This article describes the cultural overlays that are common to birth practices } \\
\text { everywhere. }\end{array}$ \\
\hline
\end{tabular}

\section{Birth, Culture and Women's Status}

Although childbirth is a universal fact of human physiology, the social nature of birth and its importance for survival ensure that this biological and intensely personal process will carry a heavy cultural overlay, resulting in wide variation in childbirth practices: where, how, with whom, and even when a woman gives birth are increasingly culturally determined. In 1908, Arnold van Gennep noted that cultures ritualize important life transitions into what he called rites of passage-of which birth is a prime example. Like all rites of passage, birth practices reflect and reveal the core values and beliefs of the culture, telling the observer a great deal about the way that culture views the world and women's place in it. Where women's status is high, a rich set of nurturant traditions tends to develop around birth; where it is low, the opposite may occur. For example, in the patriarchal Islamic society of Bangladesh, in which the status of women is low, childbirth (like menstruation) has traditionally been regarded as highly polluting. It was believed that women should give birth on dirty linens, with the baby set aside on a pile of rags until the placenta was delivered, as that was considered the moment of greatest danger; thus, rates of neonatal mortality and puerperal infections have long been high. In contrast, in matrilineal societies such as those of Milne Bay in Papua New Guinea and of Micronesia, where the status of women is high, pregnant women are pampered and nurtured. Skilled midwives administer frequent full-body massages during pregnancy and have a rich repertoire of techniques for assisting women during labor and birth.

\section{The Effects of Place of Birth}

Brigitte Jordan's (1993 \& 1978) comparative study of birthing systems in Holland, Sweden, the United States, and Mexico's Yucatan, was the first to comprehensively document the wide cultural variations in birth. Her biocultural approach used the cultural definition of birth, the place of birth, birth attendants, artifacts employed to facilitate or control birth, and differences in knowledge systems about birth as foci for cross-cultural comparison. Among these, place of birth and the beliefs and ideologies of the practitioners who attend births have emerged as the most salient for how birth happens. In home settings across cultures, from huts to houses, childbirth flows according to the natural rhythms of labor and women's social routines. In early labor, women move about at will, stopping their activities during the forty-five seconds or so per contraction, and then continuing to be active (which may include doing chores, cooking, chatting, walking, eating, singing, and dancing). Such activities subside as they begin to concentrate more on the work of birthing, often aided by massage and emotional support from their labor companions, who are usually midwives and relatives.

Many cultures have rich traditions about who should be present (sometimes the father, sometimes only women, sometimes the whole family and/or friends), how labor support should be provided, what rituals should be performed to invoke the help of Gods, Goddesses and/or ancestor spirits, and what herbs and hand 
maneuvers may be helpful to assist a birth or stop a postpartum hemorrhage. When birth is imminent, women at home usually take upright positions, squatting, sitting, standing, or on hands and knees, often pulling on a rope or pole or on the necks or arms of their companions, and work hard to give birth, rewarded by the baby in their arms. Postpartum practices vary widely: some cultures encourage early breastfeeding, whereas some code colostrum as harmful and feed the baby other fluids until the breastmilk comes in (a dangerous practice; many babies die from contaminated water). Most especially in low-resource countries, long-term breastfeeding is essential for infant survival. Steam and herbal baths, and periods of postpartum confinement are often culturally prescribed, varying in length from a few to forty days.

Where freestanding birth centers exist, whether staffed by traditional or professional midwives, the experience of birth is resonant with the experience of birthing at home-a free flow. There are usually no absolute rules for how long birth should take. As long as the mother's vital signs are good and the baby's heartbeat is relatively stable, trained attendants allow labor to proceed at its own pace, keeping their focus on the needs of the mother for emotional and physical support. Skilled midwives recognize the difference between the latent phase of labor (up to $6 \mathrm{~cm}$ of cervical dilation), which can safely and normally take many hours or even days as the uterus tones and prepares for its work of delivery, and the active phase (from $6 \mathrm{~cm}$ on), which is usually much more rapid. Birth in the hospital and or in a medical clinic is an entirely different experience. Busy techno-medically trained practitioners who either don't recognize the normality of the latent phase (during which, if possible, women should not be admitted to the hospital) or lack both the time and the patience to nurture the mother through a lengthy latent phase, tend to assume that birth should follow a certain pattern, including cervical dilation of one centimeter per hour-an arbitrary rule unsupported by science but consistent with industrial patterns of production.

Ensuring the mechanical consistency of labor requires frequent manual checking of cervical dilation, which, if determined to be proceeding too slowly, will be augmented by breaking the amniotic sac (which introduces the possibility of fetal infection) and intravenous administration of the synthetic hormone Pitocin (syntocinon) to speed labor. Women are often not allowed to eat or drink and thus are routinely hydrated through intravenous lines, which also facilitate the administration of Pitocin to speed labor and other drugs. Electronic fetal monitoring to record the strength of the mother's contractions and the baby's heartbeat is pervasive in Western-style hospitals, in spite of the fact that its routine use does not improve most birth outcomes but does significantly raise the cesarean rate-because it shows every fetal heart deceleration (most of which are normal during labor), causing practitioners to assume that the baby is in distress when it is not. Intermittent auscultation of the fetal heart rate has been proven to be more effective than electronic fetal monitoring at detecting fetal distress. Yet the monitor is highly symbolic of the supervaluation of high technologies in technocratic societies (or those striving to modernize) around the world; its constant use provides an ongoing flow of information, reflecting the high cultural value technocratic societies place on information that comes from high-tech machines such as the EFM and ultrasound, which is increasingly used to determine fetal size and position instead of the hands-on uterine palpation midwives are trained in but doctors, increasingly, are not.

Episiotomies to widen the vaginal outlet at the moment of birth are also common, although scientifically demonstrated to be unnecessary in $90 \%$ of births. Because of the preponderance of evidence against routine episiotomy, United States and European obstetricians have drastically reduced its use to around $10 \%$, while in many low-resource countries the episiotomy rate for vaginal birth is $100 \%$, because obstetricians in these countries are taught that the baby will not come out without the cut and/or, as many have noted, that "the perineum will explode." I have long interpreted such routine obstetric procedures as rituals that symbolically enact and display the core values of the technocracy, which center around an ethos of progress through the development and application of ever-higher technologies to every aspect of human life, including reproduction (Davis-Floyd 2003[1992]). Their routine overuse has been given an acronym by Miller and colleagues (2016): TMTS-"too much, too soon."

\section{Impact of the Western Technocratic Model of Birth in Low-Resource, Developing Countries}

Miller et al. contrast TMTS interventions with TLTL ("too little, too late") failures of care, in which a strong push on the part of development agencies and national ministries of health to move birthing people into medical facilities combines with inadequate staff, training, infrastructure, supplies, and medications. The alltoo-common result is care that is poor in quality, withheld, or simply unavailable until it is too late. The growing worldwide supervaluation of advanced Western technologies has induced many developing countries to destroy formerly viable indigenous birthing systems and import the Western model even when it is ill suited to the local situation. Hospital staff members often have little understanding of or respect for local birth traditions and values, with the result that local women often avoid such hospitals whenever possible. From Northern India to Papua New Guinea to Croatia to Mexico, indigenous women echo each other's concerns about biomedical hospitals and clinics in both rural and urban areas: "they expose you," "they shave you," "they cut you," "they leave you alone and ignore you, but won't let your family come in to support you and feed you," "they give you nothing to eat or drink," and "they yell at you and sometimes slap you if you do not do what they say." Such treatment reflects the importation of the culturally insensitive technocratic model and demonstrates why more and more birth activists around the world are using the terms obstetric violence, 
disrespect, and abuse to characterize such practices and are insisting that these forms of violence, whether subtle or overt, constitute violations of women's human rights.

The transglobal imposition of this technocratic model on childbirth, sold to governments as "modern health care" and to women as "managing risk" and "increasing safety in birth," has resulted in an explosion of TMTS technological interventions in birth (including cesarean sections) unprecedented in human history. Despite the World Health Organization's demonstration that, although a national cesarean rate below 10 percent means that women are getting inadequate care often resulting in maternal and/or fetal death, when the rate is higher than 15 percent, it generally means that many cesareans are neither necessary nor life-saving. In many countries, cesarean rates are above 40 percent. For example, in most Latin American countries, it is normal for private hospitals to have cesarean section rates of 80 percent or higher, with rates of 30-40 percent in public hospitals. Greece has possibly the highest national cesarean section rate in the world, at around 65-70 percent. Cesarean section rates in India and China vary widely, from 100 percent in some private urban hospitals to dangerously low in rural areas. The cesarean section rate in the United States stopped rising around 2011 and currently still stands at 32 percent, and those for United Kingdom and New Zealand at around 25 percent.

Physician convenience and economic gain, combined with deeply ingrained medical beliefs that birth is a pathological process that works best when technologically controlled, are significant factors in this cesarean epidemic, along with the de-skilling of obstetric residents, who are no longer taught how to use hand maneuvers to deliver babies in problematic positions nor how to turn them in the womb before labor begins. The World Health Organization standard is nearly met in the Netherlands, with its cesarean rate of 16 percent, and is reinforced by the excellence of birth outcomes in that country. This success is entirely cultural: the definition of birth as a normal physiologic process in the Netherlands, in combination with Dutch cultural values on family, midwifery care, and careful attention to scientific evidence, have led to minimal interventions in hospital birth and a higher homebirth rate (13 percent). In most of the developed world, homebirth rates hover around 1 percent, despite the demonstrated efficacy and safety of planned, midwifeattended home births (de Jonge et al., Cheyney et al.). Contesting Biomedical Hegemony.

\section{Contesting Biomedical Hegemony}

The global hegemony of this technocratic model is heavily contested. In addition to the thousands of local birthing systems, three primary paradigms for contemporary childbirth exist throughout the world: the technocratic, the humanistic, and the holistic models (Davis-Floyd 2018 [2001]). The highly patriarchal technocratic model of biomedicine metaphorizes women's birthing bodies as dysfunctional machines and encourages aggressive intervention in the mechanistic process of birth. The reform effort located in the humanistic model stresses that the birthing body is an organism influenced by stress and emotion and calls for relationship-centered care, respect for women's needs and desires, and a physiologic, evidence-based approach to birth. This humanistic approach can incorporate epidurals (pain-relieving analgesia that numbs the woman from the chest down), which many women want and are unlikely to cause harm if administered after active labor has kicked in. (Giving an epidural too early in labor, as often occurs in the technocratic approach, can slow labor down, thus "necessitating" the administration of Pitocin to speed it back up, in what is known as the TMTS "cascade of interventions" in which one leads to more, often resulting in an "emergency" cesarean section due to the stress to the baby from the more powerful Pitocin-induced contractions, duly registered on the monitors).

In many low-resource countries, epidurals are simply not available. In the United States, they are used in around 60 percent of births, whereas in the high-resource country of Japan, the epidural rate is only around 6 percent, as women tend to expect pain in labor and consider it to be an important part of the process of transformation into motherhood. The more radical holistic model defines the body as an energy system, stresses spiritual and intuitive approaches to birth, and places a high value on "the feminine"-without essentializing women as "slaves to their biology." Rather, in both the humanistic and holistic paradigms, women are the protagonists and the choices are theirs to make, whereas in the technocratic paradigm, women are passive recipients of whatever kind of care their practitioners choose to provide. In dozens of countries, humanistic and holistic practitioners and consumer members of growing birth activist movements are using scientific evidence and anthropological research to challenge the dominant technocratic model of birth. They seek to combine the best of indigenous and professional knowledge systems to create healthier, safer, and more cost-effective systems of birth care. Yet, from a cross-cultural point of view, all three paradigms are limited by their focus on the care of the individual.

For example, mortality resulting from parturition (pregnancy, birth, and the post-partum period) is widely recognized as a massive global problem, costing over 300,000 lives per year. Technomedicine identifies conditions such as hemorrhage and toxemia as major causes of maternal death; advises investment in doctors, hospitals, and rural clinics to provide prenatal care to prevent toxemia; and recommends active intervention immediately after birth (administration of Pitocin, and cord traction for rapid removal of the placenta-an extremely dangerous intervention) to prevent hemorrhage. This Techno-medical approach makes it appear that problems inhere in individuals and should be treated on an individual basis, patient by patient, hospital by hospital. In contrast, social science research in countries with the highest maternal mortality rates (e.g., sub-Saharan Africa) highlights the general poor health of women, who in many patriarchal societies suffer from overwork, 
exhaustion, anemia, malnutrition, and a variety of diseases resulting from polluted water, showing that the most important interventions required for improving women's health and for increasing safety in birth are clean water, adequate nutrition, and improved educational and economic opportunities for women.

\section{Back to the Future: Science and Midwifery Care}

Deep in the evolutionary past, our ancestors came to understand the benefits of women helping other women to give birth (Trevathan 1987). Today, the most successful birthing systems combine midwifery-and, increasingly, doula care-with solid scientific research on the physiology of birth, resulting in what Melissa Cheyney and I (2019) have termed the RART approach-"the right amount at the right time." Contemporary midwives and doulas (supportive labor companions) work in all settings, from hospital to home, and support women to avoid unnecessary interventions and to move around a lot during labor, as frequent movement is the essence of a healthy labor, as is eating and drinking at will to give women the strength they need). Such practitioners encourage women to give birth in upright or side-lying positions (not on their backs, as this lithotomy position compresses the pelvic outlet, making pushing more difficult), and to enjoy uninterrupted contact with their babies after birth, which facilitates breastfeeding. (See Davis-Floyd et al. for many examples of highly functional and effective birth models).

In the United States, obstetricians solidified their control over birth during the first half of the 20th century and had nearly eliminated midwifery by the 1950s. Since then the demands of many women for the more one-on-one, time-intensive, and humanistic care midwives provide have generated a small midwifery renaissance. U.S.-based research, such as Eugene Declerq's 2015 study, reports that by 2012, certified nurse-midwives (CNMs) had reached attendance at $11.3 \%$ of total U.S. births while "other" midwives, mostly certified professional midwives (CPMs), attended around $1.36 \%$-a significant rise from the $2 \%$ midwives cumulatively attended in 1979. A major midwifery renaissance has also taken place in New Zealand. As Georges and Dallenbach (2019) report, professional midwives were nearly nonexistent in New Zealand by the 1980 s, yet today, they are present at 100 percent of birthseven at scheduled cesareans performed by obstetricians; they also are chosen to serve as primary caregivers by 92 percent of pregnant women. And the long-standing midwifery systems of the Netherlands, the Scandinavian countries, and to some extent the United Kingdom continue to achieve excellent results.
The World Health Organization, the International Confederation of Midwives, and other international development organizations have issued a call for 350,000 more professional midwives to be trained in the very near future to meet the demand for skilled care and evidence-based birth practices, most especially in lowresource countries. Traditional midwives, formerly the primary care providers for the majority of women in these countries, are being intentionally phased out of practice in most of them because development agencies and Ministries of Health have come to perceive them as generally "untrainable" in best practices and thus unable to lower the still unacceptably high maternal and perinatal mortality rates in those nations. Much anthropological research addresses the faulty nature of these perceptions and stresses the lamentable fact that traditional midwives are almost never trained in the lifesaving and easy-to-learn proper administration of oxytocin injections, or of Cytotec/misoprostol, to stop post-partum hemorrhages on the ground rather than having to try to transport the bleeding mother to a faraway-and often unreachable-medical facility where TLTL care may prevail.

Yet such perceptions of "unattainability" persist to the extent that it seems clear, sadly, that traditional midwives will not accompany us into the future, in spite of their invaluable services in the present and the past. When their training and practice are overly biomedical and their work conditions stressful due to overwork and lack of essential supplies, professional midwives and nurses themselves can be the source of the problem of obstetric violence, sometimes to the same extent as doctors. In contrast, midwives and nurses trained in what is internationally known as "the midwifery model of care"-which focuses on keeping the woman at the center of care and facilitating normal physiologic birth in most cases while identifying and addressing pathology where it truly exists--are often the solution. It has repeatedly been demonstrated that practitioners of the RART midwifery model perform fewer interventions, generate less iatrogenic damage to mothers and babies, improve outcomes (both psychological and physical), and massively lower costs (Cheyney et al.; de Jonge et al.). It is to be hoped that in short order the world will pass through the current phase of high-technology interventions in what should and could be normal birth and come full spiral, uniting evolutionary understandings with contemporary science through midwives' skilled, nurturant, and woman-centered care. 
ISSN: 2574-1241

DOI: 10.26717/BJSTR.2019.20.003467

Robbie E Davis-Floyd. Biomed J Sci \& Tech Res

(C) This work is licensed under Creative

Submission Link: https://biomedres.us/submit-manuscript.php

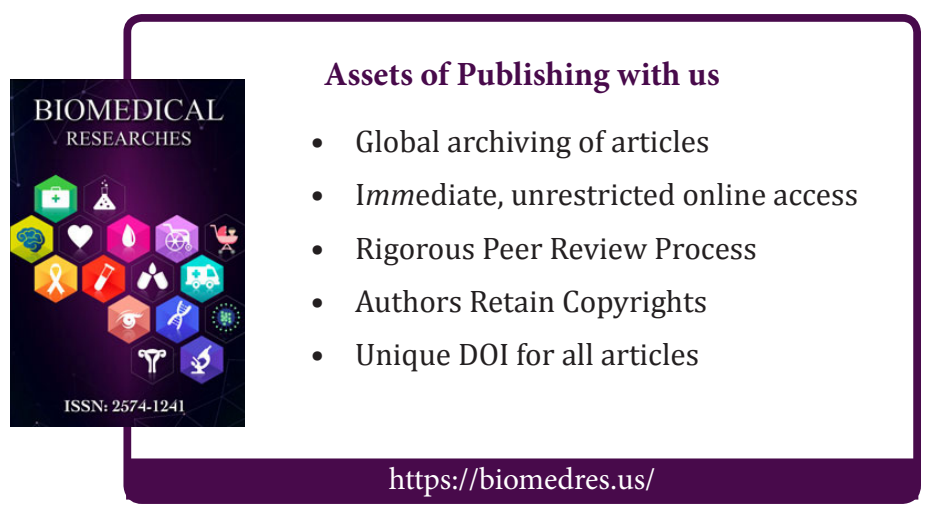

